\title{
Configuration Design of Novel Manually Operated Dynamo Flashlights*
}

\author{
Hong-Sen YAN** and Hsin-Te WANG*** \\ **Department of Mechanical Engineering, National Cheng Kung University, \\ No. 1 University Road, Tainan, Taiwan 70101, ROC \\ E-mail:hsyan@mail.ncku.edu.tw \\ *** Metal Industries Research \& Development Centre \\ 1001 Kaonan Highway, Kaohsiung, Taiwan 81160, ROC \\ E-mail:charw@mail.mirdc.org.tw
}

\begin{abstract}
This paper synthesizes novel configurations of manually operated dynamo flashlights. Topology and motion characteristics of existing gear dynamos are modified and concluded. The structural sketches and corresponding graph representations for gear trains and dynamos with the defined induced magnetic circuits are defined. Through the concepts of generalization and specialization, the atlas of the structural sketches and graphs of the embedded gear dynamos is obtained subject to the defined design requirements and constraints. And, a systematic approach is proposed to synthesize the novel mechanisms of the embedded gear dynamos. As a result, the embedded three-link and four-link gear dynamos have 12 and 24 novel design configurations, respectively. One prototype of the embedded three-link and another of the embedded four-link gear dynamo are built.
\end{abstract}

Key words: Configuration Synthesis, Dynamo, Gear Train, Graph

\section{Introduction}

In recent years, the recyclable and rechargeable mechatronics products, such as manually operated dynamo flashlights, are getting more attentions. Generally, a dynamo flashlight consists of a mechanical system and an electrical system as a mechatronics device. The mechanical system includes the gear transmission and the dynamo. The electrical system includes the electrical circuit and components. This paper intends to study the compact mechanical system that the dynamo embeds with a gear transmission. Figure 1 shows three different mechanical systems to generate the electric power for manually operated dynamo flashlights. Figure 1(a) is a shaking type and is the simplest in structure $^{(1)}$. It has no other transmission to accelerate the rotor. It is a typical experiment of the Faraday's Law. And, the other two types of dynamos in Figure 1 also apply this fundamental law to produce the induction electric voltage and current. However, the shaking type is not a continuous motion and the power output is lower. In Figure 1(b), the rotor of a dynamo is accelerated by the gear train and the generated electric energy is preserved in the storage batteries for further applications, such as lights, radios, clocks, or alarms ${ }^{(2)-(5)}$. The windup type dynamo flashlight, Figure 1(b), is incorporated with the gear train and the dynamo to charge the batteries and provide the output energy. Some patents ${ }^{(6)-(7)}$ proposed other drive methods to provide a continuous power source. The gear train transfers the low input velocity (high torque) to a stable and high relative rotation between the rotor and the stator of the dynamo. In Figure 1(c), the squeeze type dynamo flashlight was used for rechargeable apparatuses ${ }^{(8)}$. The disadvantages in the squeeze type dynamo are louder noises, higher friction losses, a longer power transfer path, and too many 
components in the system. Although the windup type dynamo has a continuous motion, it is not easy to operate in a higher input revolution. Recently, Modernoutpost ${ }^{(9)}$ demonstrated these designs and other related productions, and focused on modifying or integrating partly structures of dynamos and gear trains. So far, no studies target on the integration design of the structures of the dynamos embedded in gear trains that not only reduces the overall size and complexity of the system but also reduces the cost. Furthermore, the dynamo and the gear train are usually designed and manufactured separately. According to a systematic structural synthesis of the integrated gear motors ${ }^{(10)}$, it can also be applied on the configuration of the mechatronics, such as gear dynamos.

The purpose here is to present a design methodology for the systematic generation of all possible configurations of dynamos embedded in gear trains subject to topology and motion characteristics, Figure 2. Existing designs are studied first. Then, graph representations for the components of dynamos and gear trains are applied, novel structures of the dynamo embedded in gear trains are synthesized, and the simplified structural sketches for the structures of the integration designs are obtained. Finally, two prototype concepts are illustrated.

\section{Existing Designs}

Since Figure 1(a) shows the simplest dynamo flashlight without a transmission, the mechanical system is not discussed here. Figures $1\left(b_{2}\right)$ and $1\left(c_{2}\right)$ show the simplified sketches for a dynamo embedded in a four-link gear train. The rotor of the squeeze type dynamo is connected to gear 3 of the four-link gear train with the ratchet. The ratchet is functioned only when gear 1 (hand bar) inputs the squeeze motion. If the hand bar releases, the ratchet is not meshed with the ratchet wheel (rotor). The path of the input force and torque is transmitted from gear 1 to gear 2 , gear 3, and the rotor. And, the rotor

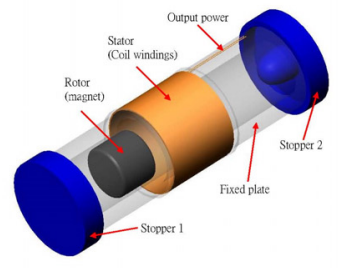

(a) Mechanism

(a) Shaking type

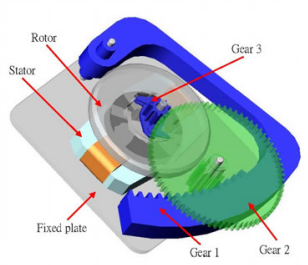

$\left(b_{1}\right)$ Mechanism

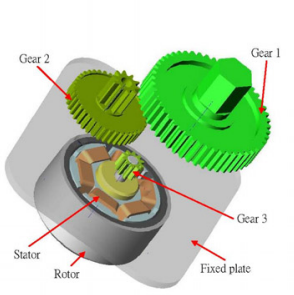

$\left(c_{1}\right)$ Mechanism $\left(a_{2}\right)$ Structure sketch
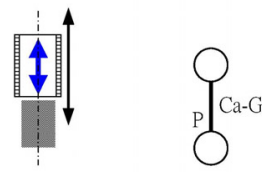

(a) Graph

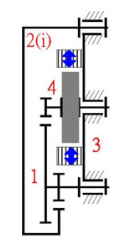

$\left(b_{2}\right)$ Structure sketch

(b) Squeeze type

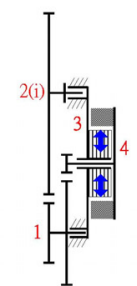

$\left(c_{2}\right)$ Structure sketch

c) Windup type

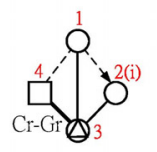

(b) Graph

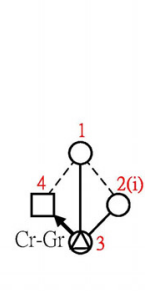

(c) Graph

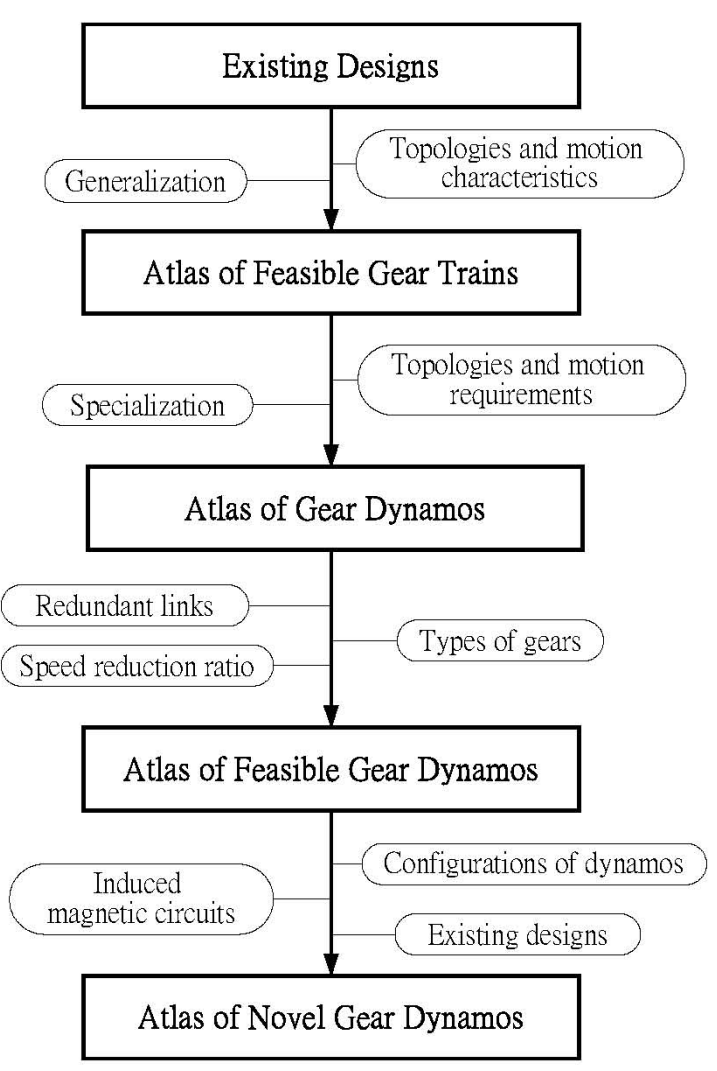

Figure 2. Process of configuration synthesis 
has a large moment of inertia to stable the input motion. In Figure 1(c), the rotor of the windup type dynamo is combined with gear 3 without the ratchet. The path of the input force and torque is the same as above. And, the rotor has no large inertia to stable the input motion.

\subsection{Topology characteristics}

By analyzing available existing designs of dynamos embedded in gear trains, their topology characteristics are concluded as follows:

1. The four-link gear train is a closed-type mechanism with one degree-of-freedom (DOF).

2. The gear train has one fixed member and one input member.

3. The joint is incident to the fixed and the input members must be a revolute joint.

4. The dynamo has a permanent magnet and a different member as the armature. Since one of the two members is fixed, it is considered as the stator; otherwise, it is the rotor.

5. The joint incident to the permanent magnet and the armature is also a revolute joint which cannot be the same joint incident to the fixed and the input members. And, it has three configurations (exterior-rotor, interior-rotor, and axial-gap types) in dynamos.

6. A copper brush must be constructed on the rotating armature for transmitting the electrical current. Otherwise, the brush is not necessary in the fixed armature. Since the brush is not considered as the main component of the dynamo, it is ignored in the configuration synthesis process.

7. The numbers of the poles in permanent magnets and armatures are not considered in the design process, since they do not affect the structure of the dynamos. Furthermore, the numbers of external and internal gear teeth in the gear train are also not considered.

\subsection{Motion characteristics}

Based on existing design, the motion characteristics of dynamos embedded in gear trains are concluded as follows:

1. As long as the permanent magnet retains a relative rotation with the armature, the dynamo still can generate the electricity through the coil windings. Therefore, the configuration of two rotors with relative rotation is available.

2. The induced magnetic circuit is constructed on the joint incident to the permanent magnet and the armature and alternatively varied direction with the corresponding induced magnetic field. And, the induced electric circuit is generated in the coil windings as the alternative induced magnetic circuit varied.

3. The directions of coil windings in the armatures and the directions of air gaps between the permanent magnets and armatures establish the induced magnetic circuit. They greatly affect the output power of dynamos. However, only radial or axial directions are considered in this work. And, their directions can be varied. Therefore, there are four combinations of induced magnetic circuits in dynamos.

\section{Atlas of Feasible Gear Trains}

Traditionally, graphs are applied to represent the topological structures of gear trains. However, edges and vertices of graphs ${ }^{(11)-(15)}$ are not enough to represent the configurations of gear trains and dynamos. Here, the edges are symbolized as a dash line and a thin line that represent a gear joint and a simple revolute joint incident to two links, respectively. The vertices are symbolized as a circle, a concentric circle, a square, and a triangle within a circle to represent a moving member, a fixed member, a rotor, and a stator, respectively. A crosshatched triangle represents a multiple revolute joint incident to three links. An external gear joint is represented as a dash line and an internal gear joint is represented as a directional dash line. Table 1 summarizes the definitions of the graph representations for all components in gear trains and dynamos. 
Existing dynamos can be classified as five cases as shown in Figure 3. A thick line, a unidirectional thick line, and a bidirectional thick line between the rotor and the stator indicate an interior-rotor, an exterior-rotor, and an axial-gap type configuration, respectively. In general, the existing dynamos have a rotor as the moving member and a stator as the fixed member. However, based on the motion characteristics (1), since one rotor within the permanent magnet keeps the relative rotation to the other rotor within the armature, the dynamo is still functioned. Therefore, three novel graphs of two rotors with relative rotation in each dynamo are shown in Table 2.

Table 1. Graph representations of components in gear dynamos ${ }^{(10)}$

\begin{tabular}{|c|c|c|c|c|}
\hline Graphs & Definitions & \multirow{3}{*}{\multicolumn{3}{|c|}{ Table 2. Three configurations of the revolute joint in dynamos }} \\
\hline & Gear joint & & & \\
\hline & Simple revolute joint & & & \\
\hline & Multiple revolute joint & \multirow{2}{*}{ Configurations } & \multirow{2}{*}{ Graphs } & \multirow{2}{*}{ Definitions } \\
\hline \multirow[b]{2}{*}{ (2) } & Moving member & & & \\
\hline & Fixed member & \multirow{2}{*}{$\begin{array}{l}\text { Interior-rotor } \\
\text { type }\end{array}$} & & Stator and rotor \\
\hline & Internal gear & & & $\begin{array}{l}\text { Two rotors with relative } \\
\text { rotation }\end{array}$ \\
\hline & External gear & \multirow{3}{*}{$\begin{array}{c}\text { Exterior-rotor } \\
\text { type }\end{array}$} & & \\
\hline$--\infty$ & Fixed internal gear & & & Stator and rotor \\
\hline-- & Fixed external gear & & & $\begin{array}{l}\text { Two rotors with relative } \\
\text { rotation }\end{array}$ \\
\hline \multirow[b]{2}{*}{$\theta$} & Rotor & \multirow{3}{*}{ Axial-gap type } & & Suts det \\
\hline & Stator & & & Stator and rotor \\
\hline-- & Rotor within an external gear & & & $\begin{array}{l}\text { Two rotors with relative } \\
\text { rotation }\end{array}$ \\
\hline--- & Rotor within an internal gear & & & \\
\hline$--\infty$ & Stator within an external gear & & & \\
\hline$--\theta$ & Stator within an internal gear & & & \\
\hline
\end{tabular}

The process of generalization is based on a set of generalizing rules ${ }^{(13)}$. For the graphs of embedded gear dynamos shown in Figures $1\left(b_{1}\right)$ and $1\left(c_{1}\right)$, the steps of generalization are as follows:

1. The fixed link is released.

2. The released fixed link and the input link are generalized into binary or ternary links.

3. The joints are generalized into simple or multiple revolute joints.

4. The members of dynamos are generalized into binary links.

5. The joints of internal gears are generalized into external gear joints

6. The joints of dynamos are generalized into revolute joints.

7. The labeled induced magnetic circuits are generalized without labels.

For the two designs shown in Figures $1\left(b_{3}\right)$ and $1\left(c_{3}\right)$, the corresponding generalized graphs are shown as Figure $4\left(a_{1}\right)$. And, Figures $4\left(a_{2}\right)$ and $4\left(a_{3}\right)$ are the corresponding atlas of graphs for the one DOF four-link gear trains.

\section{Atlas of Embedded Gear Dynamos}

For simplification, an embedded dynamo in a gear train is called the embedded gear dynamo for short. In order to synthesize all possible configurations of the four-link embedded gear dynamos, the existing mechanisms are transformed into generalized graphs. Then, the extended feasible generalized graphs are specialized subject to topology and motion requirements to obtain the embedded four-link gear dynamos.

The process of specialization is to assign specific types of members and joints into the available atlas of generalized graphs to have all possible specialized graphs subject to topology and motion requirements ${ }^{(13)}$. The topology and motion requirements are 
concluded as follows:

1. The input link cannot be the fixed link.

2. The fixed link and the input link are incident to the revolute joint.

3. The two members of the dynamo must be incident to another revolute joint.

4. One of the two members of the dynamo can be the input or the fixed link.

Now, take the four-link one DOF gear trains as shown in Figure 4( $\left.a_{1}\right)$ for example. By assigning the fixed and the input links respectively, there are four graphs as shown in Figures $4\left(b_{1}\right), 4\left(b_{2}\right), 4\left(b_{3}\right)$, and $4\left(b_{4}\right)$. The input link has the label (i) on its link number. Then, by assigning the two members of the dynamo to Figure $4\left(b_{1}\right)$ corresponding graph $4 \mathrm{~A}$ in Figure 4(c) is obtained.

Table 3. Graph representations of induced magnetic circuit in dynamos

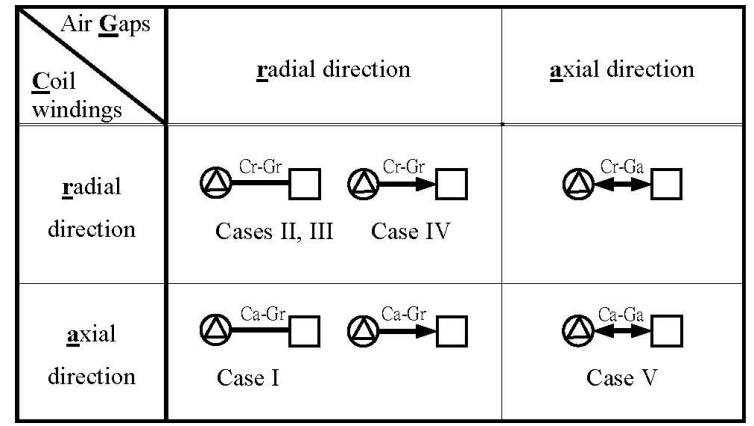

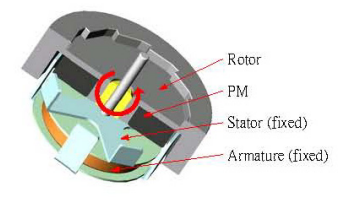

(a) Case I (interior-rotor type)

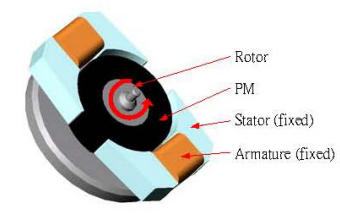

(b) Case II (interior-rotor type)

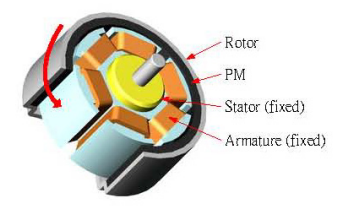

(d) Case IV (exterior-rotor type)

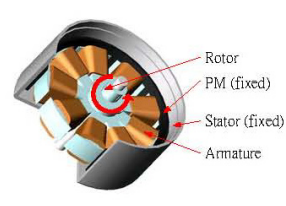

(c) Case III (interior-rotor type)

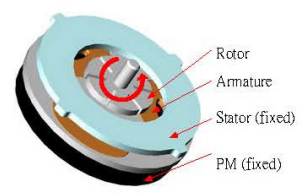

(e) Case V (axial-gap type)

Figure 3. Structures and components of existing dynamos

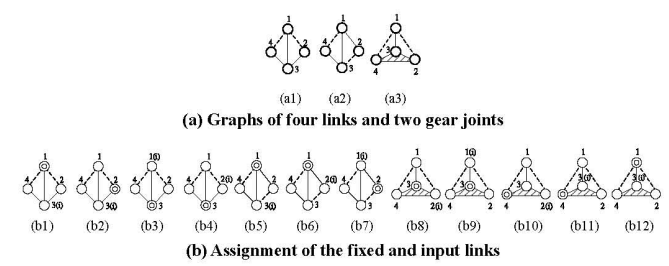

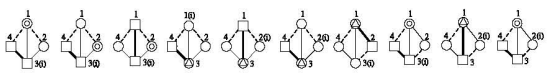
$\begin{array}{cccccccccc}4 \mathrm{~A} & 4 \mathrm{~B} & 4 \mathrm{C} & 4 \mathrm{D} & 4 \mathrm{E} & 4 \mathrm{~F} & 4 \mathrm{G} & 4 \mathrm{H} & 4 \mathrm{I} & 4 \mathrm{~J} \\ 4 & 4 & 1 & 1 & 1 & 10 & 40 & 1 & 1 & 1\end{array}$

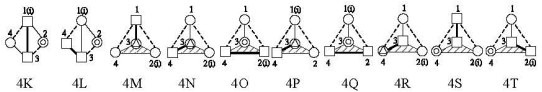

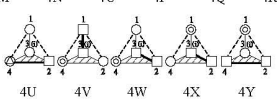

(c) Assignment of the two mem bers of dynamos

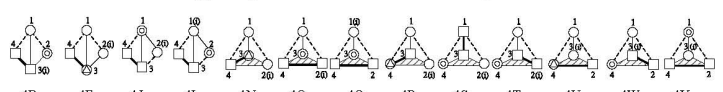

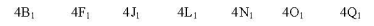

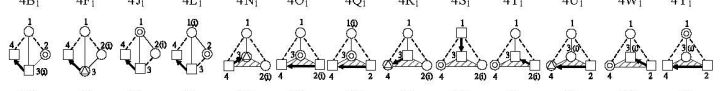
$\begin{array}{lllllllllllll}4 \mathrm{~B}_{2} & 4 \mathrm{~F}_{2} & 4 \mathrm{~J}_{2} & 4 \mathrm{~L}_{2} & 4 \mathrm{~N}_{2} & 4 \mathrm{O}_{2} & 4 \mathrm{Q}_{2} & 4 \mathrm{R}_{2} & 4 \mathrm{~S}_{2} & 4 \mathrm{~T}_{2} & 4 \mathrm{U}_{2} & 4 \mathrm{~W}_{2} & 4 \mathrm{Y}_{2}\end{array}$

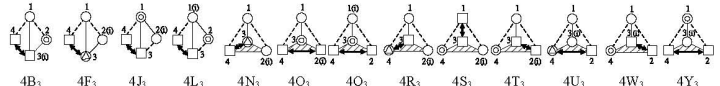
(d) Assignment of the three configurations of dynamos

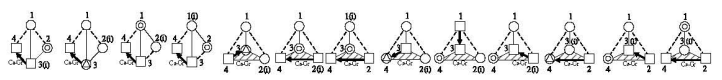

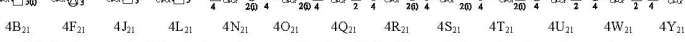
(e) Assignment of the induced magnetic circuit $\mathrm{Ca}-\mathrm{Gr}$ on internal rotor of gear dynamos

Figure 4. Configurations of the embedded four-link gear dynamos 


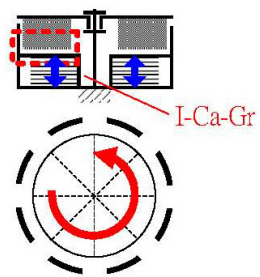

(a) Case I
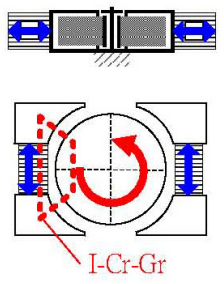

(b) Case II

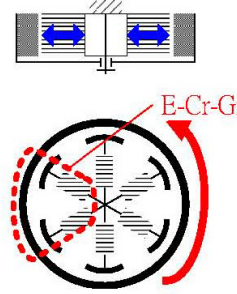

(d) Case IV

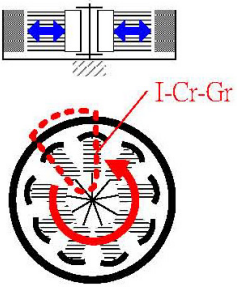

(c) Case III

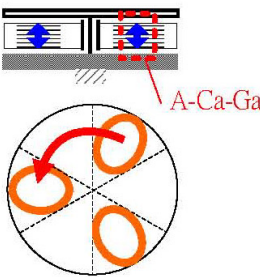

(e) Case V

Furthermore, the radial and axial directions for the coil windings on the armatures and air gaps between the permanent magnet and the armature are defined to express the induced magnetic circuit as shown in Figure 5. Labels I, E, and A represent the interior-rotor, exterior-rotor, and axial-gap type configuration of dynamos, respectively. Labels $\mathrm{C}$ and $\mathrm{G}, \mathrm{Cr}$ and $\mathrm{Ca}$, and $\mathrm{Gr}$ and $\mathrm{Ga}$ are defined as coil windings and air gaps, the radial and axial directions of the coil windings, and the radial and axial directions of air gaps, respectively. Table 3 illustrates all six labels of the induced magnetic circuits with the corresponding cases in Figure 5. The I-Ca-Gr of the induced magnetic circuit within dynamo is illustrated such that the dynamo is the interior-rotor type configuration with axial direction of coil windings and radial direction of air gaps

As a result, each configuration in

Figure 5. Induced magnetic circuits of existing dynamos

Table 3 has two kinds of the induced magnetic circuits. The interior-rotor, exterior-rotor, and axial-gap type configurations have the induced magnetic circuits of $\mathrm{Cr}-\mathrm{Gr}$ and $\mathrm{Ca}-\mathrm{Gr}$, $\mathrm{Cr}-\mathrm{Gr}$ and $\mathrm{Ca}-\mathrm{Gr}$, and $\mathrm{Cr}-\mathrm{Ga}$ and $\mathrm{Ca}-\mathrm{Ga}$, respectively. Hence, the dynamos have to arrange the two components: permanent magnets and armatures on the above three configurations after the assignment of the related induced magnetic circuits. In addition, if the armature is the moving member and generates the electricity to the fixed bulb, the brush should append on the rotor. However, the brush is neglected and is not one of the two members in the dynamo during the process of configuration synthesis.

Table 4. Redundant links of the four-link graphs in Figure 4(c)

\begin{tabular}{|c|c|c|c|}
\hline Graphs & Sets of fundamental circuits & Set $\{C\}$ & $\begin{array}{l}\text { Redundant } \\
\text { links }\end{array}$ \\
\hline $4 \mathrm{~A}$ & $\begin{array}{l}\left\{V_{1}\right\}=\{1,2,3\},\{1,3,4\} \\
\left\{V_{2}\right\}=\{1,2,3,4\}\end{array}$ & $\{1,3,4\}$ & 2 \\
\hline $4 \mathrm{~B}$ & \multirow{2}{*}{$\begin{array}{l}\left\{\mathrm{V}_{1}\right\}=\{1,2,3\},\{1,3,4\} \\
\left\{\mathrm{V}_{2}\right\}=\{1,2,3,4\}\end{array}$} & $\{2,3,4\}$ & None \\
\hline $4 \mathrm{C}$ & & $\{1,2,3\}$ & 4 \\
\hline $4 \mathrm{D}$ & $\begin{array}{l}\left\{\mathrm{V}_{1}\right\}=\{1,2,3\},\{1,3,4\} \\
\left\{\mathrm{V}_{2}\right\}=\{1,2,3,4\}\end{array}$ & $\{1,3,4\}$ & 2 \\
\hline $4 \mathrm{E}$ & \multirow{2}{*}{$\begin{array}{l}\left\{\mathrm{V}_{1}\right\}=\{1,2,3\},\{1,3,4\} \\
\left\{\mathrm{V}_{2}\right\}=\{1,2,3,4\}\end{array}$} & $\{1,2,3\}$ & 4 \\
\hline $4 \mathrm{~F}$ & & $\{2,3,4\}$ & None \\
\hline $4 \mathrm{G}$ & \multirow{2}{*}{$\begin{aligned}\left\{\mathrm{V}_{1}\right\} & =\{1,2,3\},\{1,3,4\} \\
\left\{\mathrm{V}_{2}\right\} & =\{1,2,3,4\}\end{aligned}$} & $\{1,2,3\}$ & 4 \\
\hline $4 \mathrm{H}$ & & $\{1,3,4\}$ & 2 \\
\hline $4 \mathrm{I}$ & \multirow{2}{*}{$\begin{array}{l}\left\{V_{1}\right\}=\{1,2,3\},\{1,3,4\} \\
\left\{V_{2}\right\}=\{1,2,3,4\}\end{array}$} & $\{1,2,3\}$ & 4 \\
\hline $4 \mathrm{~J}$ & & $\{1,2,3,4\}$ & None \\
\hline $4 \mathrm{~K}$ & \multirow{2}{*}{$\begin{array}{l}\left\{V_{1}\right\}=\{1,2,3\},\{1,3,4\} \\
\left\{V_{2}\right\}=\{1,2,3,4\}\end{array}$} & $\{1,2,3\}$ & 4 \\
\hline $4 \mathrm{~L}$ & & $\{1,2,3,4\}$ & None \\
\hline $4 \mathrm{M}$ & \multirow{3}{*}{$\begin{array}{l}\left\{V_{1}\right\}=\{1,2,3\},\{1,3,4\} \\
\left\{V_{2}\right\}=\{1,2,3,4\}\end{array}$} & $\{1,2,3\}$ & 4 \\
\hline $4 \mathrm{~N}$ & & $\{2,3,4\}$ & None \\
\hline 40 & & $\{2,3,4\}$ & None \\
\hline $4 \mathrm{P}$ & \multirow{2}{*}{$\begin{array}{l}\left\{\mathrm{V}_{1}\right\}=\{1,2,3\},\{1,3,4\} \\
\left\{\mathrm{V}_{2}\right\}=\{1,2,3,4\}\end{array}$} & $\{1,3,4\}$ & 2 \\
\hline $4 \mathrm{Q}$ & & $\{1,2,3,4\}$ & None \\
\hline $4 \mathrm{R}$ & \multirow{3}{*}{$\begin{array}{l}\left\{V_{1}\right\}=\{1,2,3\},\{1,3,4\} \\
\left\{V_{2}\right\}=\{1,2,3,4\}\end{array}$} & $\{2,3,4\}$ & None \\
\hline $4 \mathrm{~S}$ & & $\{1,2,3,4\}$ & None \\
\hline $4 \mathrm{~T}$ & & $\{2,3,4\}$ & None \\
\hline $4 \mathrm{U}$ & \multirow{3}{*}{$\begin{array}{l}\left\{\mathrm{V}_{1}\right\}=\{1,2,3\},\{1,3,4\} \\
\left\{\mathrm{V}_{2}\right\}=\{1,2,3,4\}\end{array}$} & $\{2,3,4\}$ & None \\
\hline $4 \mathrm{~V}$ & & $\{1,3,4\}$ & 2 \\
\hline $4 \mathrm{~W}$ & & $\{2,3,4\}$ & None \\
\hline $4 \mathrm{X}$ & \multirow{2}{*}{$\begin{array}{l}\left\{V_{1}\right\}=\{1,2,3\},\{1,3,4\} \\
\left\{V_{2}\right\}=\{1,2,3,4\}\end{array}$} & $\{1,3,4\}$ & 2 \\
\hline $4 \mathrm{Y}$ & & $\{1,2,3,4\}$ & None \\
\hline
\end{tabular}

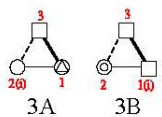

(a) Elimination of the redundant links

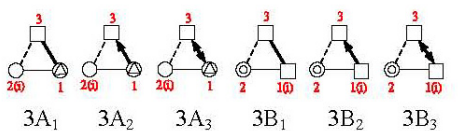

(b) Assignment of the three configurations of rotors

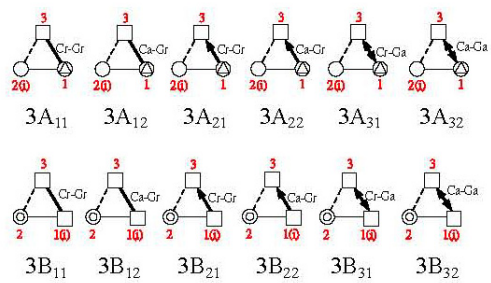

(c) Assignment of the induced magnetic circuits on each rotor

Figure 6. Configurations of the embedded three-link gear dynamos 


\section{Atlas of Feasible Embedded Gear Dynamos}

In order to obtain the atlas of feasible integration designs, the redundant links and the decelerated ones are eliminated in the atlas of integration designs. Since the redundant links do not affect the input and the output motion of the mechanism, they are neglected in the process. The speed reduction ratios of all the embedded four-link gear dynamos are evaluated after the assignment of internal and external gears. The feasible embedded four-link gear dynamos are the mechanism that can be accelerated.

\subsection{Elimination of redundant links}

The steps to analyze the redundant links in one DOF embedded gear dynamos with G gear joints are as follows:

1. List link numbers of each corresponding fundamental circuit in the embedded gear dynamo.

2. For each unrepeated set of any two, three, ..., and G-1 fundamental circuits, list the corresponding set assigned as $\left\{\mathrm{V}_{2}\right\},\left\{\mathrm{V}_{3}\right\}, \ldots$, and $\left\{\mathrm{V}_{\mathrm{G}-1}\right\}$, respectively.

3. List the unrepeated set of link numbers $\{C\}$, including the fixed link, the output link, and the two members of dynamos.

4. From the results of Step 2 and Step 3 , the sets $\left\{\mathrm{V}_{2}\right\},\left\{\mathrm{V}_{3}\right\}, \ldots$, and $\left\{\mathrm{V}_{\mathrm{G}-2}\right\}$ include set $\{\mathrm{C}\}$, and the embedded gear dynamo has redundant links. There are no redundant links in the embedded gear dynamo until the set $\left\{\mathrm{V}_{\mathrm{G}-1}\right\}$ includes set $\{\mathrm{C}\}$.

Take an embedded four-link gear dynamo, graph 4A in Figure 4(c), as an example. There are two fundamental circuits, i.e., 1-2-3 and 1-3-4. Then, the sets of link numbers $\left\{V_{1}\right\}$ are $\{1,2,3\}$ and $\{1,3,4\}$. The set $\{C\}$ is $\{1,3,4\}$ since the fixed link (stator) is link 1 , the input link is link 3 , and the rotor is link 4 . Therefore, there is a redundant link (link 2) in this embedded four-link gear dynamo. Now, take another embedded four-link gear dynamo, graph 4B in Figure 5(c), as an example. There are two fundamentals circuits, i.e., 1-2-3 and 1-3-4. Then, the sets of link numbers $\left\{\mathrm{V}_{1}\right\}$ are $\{1,2,3\}$ and $\{1,3$, $4\}$. The set $\{C\}$ is $\{2,3,4\}$ since the fixed link (stator) is link 2, the input link is link 3 , and the rotor is link 4, respectively. And, the two sets of $\left\{\mathrm{V}_{1}\right\}$ do not include the set $\{\mathrm{C}\}$. So, there is no redundant link in this embedded four-link gear dynamo. Table 4 shows the analysis of redundant analysis in all the embedded four-link gear dynamos. And, Figure 6(a) shows two three-link gear dynamos after removing the redundant links in the embedded four-link gear dynamos.

\subsection{Speed reduction ratios of the embedded four-link gear dynamos}

The atlas of feasible embedded three-link gear dynamos is obtained by assigning types of gear joints, external or internal, to the atlas of embedded four-link gear dynamos. Due to a larger moment of inertia in the internal gear, it should be assigned as the fixed link. For practical applications, the gear joints are all designated as external gears for easily analysis.

The fundamental-circuit equation of a gear pair is applied to the speed reduction ratio of a gear train in terms of the numbers of corresponding gear teeth. The fundamental circuit equation of a gear pair is ${ }^{(15)}$ :

$$
\frac{\omega_{i}-\omega_{k}}{\omega_{j}-\omega_{k}}= \pm \frac{N_{j}}{N_{i}}
$$

where $\omega_{i}, \omega_{j}$, and $\omega_{k}$ denote the angular speeds of link $i, j$, and $k$, respectively. $\quad N_{i}$ and $N_{j}$ denote the numbers of teeth on gear $i$ and $j$, respectively. The sign in Eq. (1) is positive if a positive rotation of gear $i$ relative to the carrier $k$ produces a positive rotation of gear $j$, otherwise it is negative.

The speed reduction ratio of a gear train is the ratio of output and input speed:

$$
\text { Speed reduction ratio }=\left|\frac{\omega_{\text {output }}}{\omega_{\text {input }}}\right|
$$


where $\omega_{\text {input }}$ is the angular speed of the input link and $\omega_{\text {output }}$ is the relative angular speed of the two members of the dynamo. Here, Eq. (2) is focused on its value without considering the direction of rotation. As the speed reduction ratio is greater or lesser than unity, the result of Eq. (2) shows that the mechanism can be accelerated or decelerated, respectively. Taking graph $3 \mathrm{~A}$ in Table 5 as an example, the speed reduction ratio cannot be clearly identified since the number of external gear teeth $N_{3}$ may be greater or less than the number of external gear teeth $N_{2}$. The motion of this embedded gear dynamo can be an accelerated or decelerated motion. For graph $3 \mathrm{~B}$ in Table 5, the accelerated embedded gear dynamo can be identified that the external gear has a greater number $\left(N_{2}\right)$ of teeth than the other $\left(N_{3}\right)$ at this design step. Table 5 summarizes the fundamental-circuit equations and provides speed reduction ratios of the atlas of embedded three-link gear dynamos. As a result, there are two graphs (3A and 3B) as shown in Figure 6(a) of compact and decelerating embedded Table 5. Kinematic analysis of three-link graphs in Figure 6(a) three-link gear dynamos. Furthermore, graph $3 \mathrm{~A}$ in Figure 6(a) is the embedded three-link gear dynamo with a rotor and a stator. And, graph $3 \mathrm{~B}$ in Figure 6(a) is the embedded three-link

\begin{tabular}{|c|c|c|c|}
\hline Graphs & $\begin{array}{c}\text { Fundamental circuit } \\
\text { equations }\end{array}$ & $\begin{array}{c}\text { Speed reduction } \\
\text { ratios }\end{array}$ & $\begin{array}{c}\text { Deceleration (D) } \\
\text { Acceleration (A) }\end{array}$ \\
\hline \hline 3A & $\frac{\omega_{3}-\omega_{1}}{\omega_{2}-\omega_{1}}=-\frac{N_{2}}{N_{3}}$ & $\left|\frac{\omega_{3}-\omega_{1}}{\omega_{2}}\right|=\left|\frac{N_{2}}{N_{3}}\right|$ & D or A \\
\hline 3B & $\frac{\omega_{3}-\omega_{1}}{\omega_{2}-\omega_{1}}=-\frac{N_{2}}{N_{3}}$ & $\left|\frac{\omega_{3}-\omega_{1}}{\omega_{1}}\right|=\left|\frac{N_{2}}{N_{3}}\right|$ & D or A \\
\hline
\end{tabular}
gear dynamos with two rotors.

Taking graph 4B in Table 6 as an example, the speed reduction ratio is obtained based on the numbers of gear teeth $N_{2}$ and $N_{4}$. If the number of external gear teeth $N_{4}$ is greater than $N_{2}$, the mechanism is decelerated. Otherwise, the mechanism is accelerated. And, taking graph $4 \mathrm{O}$ shown in Figure 5(c) as another example, the mechanism can be decelerated if the number of gear teeth $N_{4}$ is greater than the number of gear teeth $N_{2}$. Following the same procedures, all possible four-link results are listed in Table 6.

Table 6. Kinematic equations of four-link graphs in Figure 4(c)

\begin{tabular}{|c|c|c|c|c|}
\hline \multirow{2}{*}{$\begin{array}{c}\text { Graphs } \\
4 \mathrm{~B}\end{array}$} & \multicolumn{2}{|c|}{ Fundamental circuit equations } & \multirow{2}{*}{$\frac{\begin{array}{c}\text { Speed reduction } \\
\text { ratios }\end{array}}{\left|\frac{\omega_{4}-\omega_{3}}{\omega_{3}}\right|=\left|\frac{N_{2}}{N_{4}}\right|}$} & \multirow{2}{*}{$\begin{array}{c}\begin{array}{c}\text { Deceleration (D) } \\
\text { Acceleration (A) }\end{array} \\
\text { D or A }\end{array}$} \\
\hline & $\frac{\omega_{1}-\omega_{3}}{\omega_{4}-\omega_{3}}=-\frac{N_{4}}{N_{1}}$ & $\frac{\omega_{2}-\omega_{3}}{\omega_{1}-\omega_{3}}=-\frac{N_{1}}{N_{2}}$ & & \\
\hline $4 \mathrm{~F}$ & $\frac{\omega_{1}-\omega_{3}}{\omega_{4}-\omega_{3}}=-\frac{N_{4}}{N_{1}}$ & $\frac{\omega_{2}-\omega_{3}}{\omega_{1}-\omega_{3}}=-\frac{N_{1}}{N_{2}}$ & $\left|\frac{\omega_{4}-\omega_{3}}{\omega_{2}}\right|=\left|\frac{N_{2}}{N_{4}}\right|$ & $\mathrm{D}$ or $\mathrm{A}$ \\
\hline $4 \mathrm{~J}$ & $\frac{\omega_{3}-\omega_{1}}{\omega_{2}-\omega_{1}}=-\frac{N_{2}}{N_{3}}$ & $\frac{\omega_{4}-\omega_{3}}{\omega_{1}-\omega_{3}}=-\frac{N_{1}}{N_{4}}$ & $\left|\frac{\omega_{4}-\omega_{3}}{\omega_{2}}\right|=\left|\frac{N_{1} N_{2}}{N_{3} N_{4}}\right|$ & $\mathrm{D}$ or $\mathrm{A}$ \\
\hline $4 \mathrm{~L}$ & $\frac{\omega_{3}-\omega_{1}}{\omega_{2}-\omega_{1}}=-\frac{N_{2}}{N_{3}}$ & $\frac{\omega_{4}-\omega_{3}}{\omega_{1}-\omega_{3}}=-\frac{N_{1}}{N_{4}}$ & $\left|\frac{\omega_{4}-\omega_{3}}{\omega_{1}}\right|=\left|\frac{N_{1} N_{2}}{N_{3} N_{4}}\right|$ & $D$ or $A$ \\
\hline $4 \mathrm{~N}$ & $\frac{\omega_{1}-\omega_{3}}{\omega_{2}-\omega_{3}}=-\frac{N_{1}}{N_{1}}$ & $\frac{\omega_{4}-\omega_{3}}{\omega_{1}-\omega_{3}}=-\frac{N_{1}}{N_{4}}$ & $\left|\frac{\omega_{4}-\omega_{3}}{\omega_{2}}\right|=\left|\frac{N_{4}}{N_{2}}\right|$ & $D$ or $A$ \\
\hline 40 & $\frac{\omega_{1}-\omega_{3}}{\omega_{2}-\omega_{3}}=-\frac{N_{2}}{N_{1}}$ & $\frac{\omega_{4}-\omega_{3}}{\omega_{1}-\omega_{3}}=-\frac{N_{1}}{N_{4}}$ & $\left|\frac{\omega_{4}-\omega_{2}}{\omega_{2}}\right|=\left|\frac{N_{4}-N_{2}}{N_{4}}\right|$ & $\mathrm{D}$ or $\mathrm{A}$ \\
\hline $4 Q$ & $\frac{\omega_{1}-\omega_{3}}{\omega_{2}-\omega_{3}}=-\frac{N_{2}}{N_{1}}$ & $\frac{\omega_{4}-\omega_{3}}{\omega_{1}-\omega_{3}}=-\frac{N_{1}}{N_{4}}$ & $\left|\frac{\Phi_{1}-\omega_{2}}{\omega_{1}}\right|=\left|\frac{\mid N_{1} N_{4}-N N_{2} N_{2}}{N_{2} N_{t}}\right|$ & $D$ or $A$ \\
\hline $4 \mathrm{R}$ & $\frac{\omega_{1}-\omega_{3}}{\omega_{2}-\omega_{3}}=-\frac{N_{2}}{N_{1}}$ & $\frac{\omega_{4}-\omega_{3}}{\omega_{1}-\omega_{3}}=-\frac{N_{1}}{N_{4}}$ & $\left|\frac{\omega_{4}-\omega_{3}}{\omega_{2}}\right|=\left|\frac{N_{2}}{N_{4}-N_{2}}\right|$ & $\mathrm{D}$ or $\mathrm{A}$ \\
\hline $4 \mathrm{~S}$ & $\frac{\omega_{1}-\omega_{3}}{\omega_{2}-\omega_{3}}=-\frac{N_{2}}{N_{1}}$ & $\frac{\omega_{4}-\omega_{3}}{\omega_{1}-\omega_{3}}=-\frac{N_{1}}{N_{4}}$ & 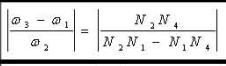 & $\mathrm{D}$ or $\mathrm{A}$ \\
\hline $4 \mathrm{~T}$ & $\frac{\omega_{1}-\omega_{3}}{\omega_{2}-\omega_{3}}=-\frac{N_{2}}{N_{1}}$ & $\frac{\omega_{4}-\omega_{3}}{\omega_{1}-\omega_{3}}=-\frac{N_{1}}{N_{4}}$ & $\left|\frac{\omega_{3}-\omega_{2}}{\omega_{2}}\right|=\left|\frac{N_{4}}{N_{2}-N_{4}}\right|$ & $\mathrm{D}$ or $\mathrm{A}$ \\
\hline $4 \mathrm{U}$ & $\frac{\omega_{1}-\omega_{3}}{\omega_{2}-\omega_{3}}=-\frac{N_{2}}{N_{1}}$ & $\frac{\omega_{4}-\omega_{3}}{\omega_{1}-\omega_{3}}=-\frac{N_{1}}{N_{4}}$ & $\left|\frac{\omega_{2}-\omega_{4}}{\omega_{3}}\right|=\left|\frac{N_{2}-N_{4}}{N_{2}}\right|$ & $D$ or $A$ \\
\hline $4 W$ & $\frac{\omega_{1}-\omega_{3}}{\omega_{2}-\omega_{3}}=-\frac{N_{2}}{N_{1}}$ & $\frac{\omega_{4}-\omega_{3}}{\omega_{1}-\omega_{3}}=-\frac{N_{1}}{N_{4}}$ & $\left|\frac{\omega_{3}-\omega_{2}}{\omega_{3}}\right|=\left|\frac{N_{4}}{N_{2}}\right|$ & D or $A$ \\
\hline $4 \mathrm{Y}$ & $\frac{\omega_{1}-\omega_{3}}{\omega_{2}-\omega_{3}}=-\frac{N_{2}}{N_{1}}$ & $\frac{\omega_{4}-\omega_{3}}{\omega_{1}-\omega_{3}}=-\frac{N_{1}}{N_{4}}$ & $\left|\frac{\omega_{4}-\omega_{2}}{\omega_{3}}\right|=\left|\frac{N_{1} N_{2}-N_{1} N_{4}}{N_{4} N_{2}}\right|$ & $\mathrm{D}$ or $\mathrm{A}$ \\
\hline
\end{tabular}




\section{Atlas of Novel Embedded Gear Dynamos}

Three configurations of the revolute joint in dynamo are assigned to the atlas of feasible embedded four-link gear dynamos. Then, four types of induced magnetic circuits are labeled on the same revolute joint for each configuration. Finally, by removing the existing designs in the synthesized results, the novel design configurations are obtained.

\subsection{Three configurations of the revolute joint in the dynamos}

Three configurations of the dynamos are interior-rotor, exterior-rotor, and axial-gap types. They are assigned to the revolute joint in dynamos. Take graph 4B in Figure 4(c) as an example, three configurations of dynamos are assigned and obtained as graphs $4 \mathrm{~B}_{1}$, $4 \mathrm{~B}_{2}$, and $4 \mathrm{~B}_{3}$ shown in Figure $4(\mathrm{~d})$. Following the same procedure, there are $39(=13 \times 3)$ four-link graphs as shown in Figure 4(d). For another three-link example, three configurations are assigned to graph $3 \mathrm{~A}$ in Figure $6(\mathrm{a})$ and three graphs $3 \mathrm{~A}_{1}, 3 \mathrm{~A}_{2}$, and $3 \mathrm{~A}_{3}$ are obtained as shown in Figure 6(b). Following the same procedure, there are six three-link graphs as shown in Figure 6(b).

Table 7. Structural representations of components in dynamos

\begin{tabular}{|c|l|}
\hline $\begin{array}{c}\text { Structural } \\
\text { representations }\end{array}$ & Definitions \\
\hline & Fixed link \\
\hline & Permanent magnet \\
\hline & Armature \\
\hline$\leftrightarrow$ & Direction of coil windings \\
\hline
\end{tabular}

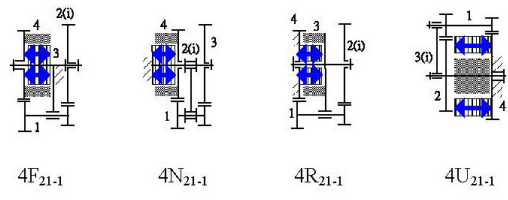

(a) Brushless gear dynamo
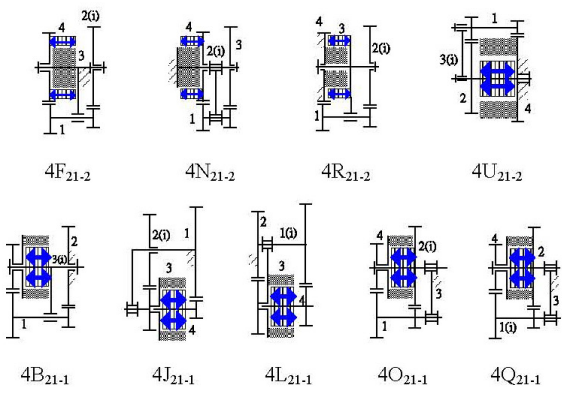

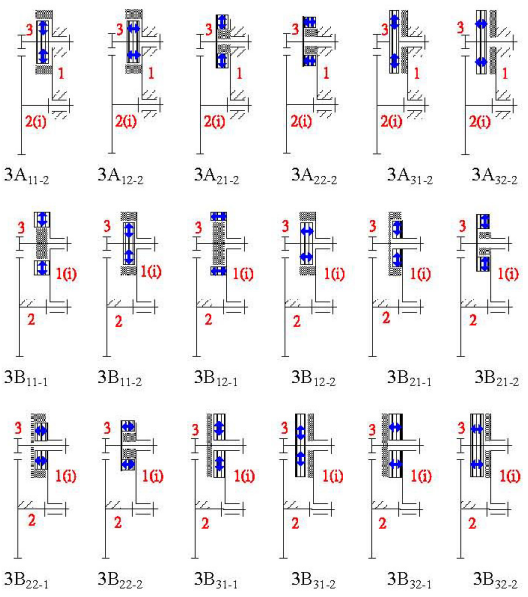

(b) Brush gear dynamos

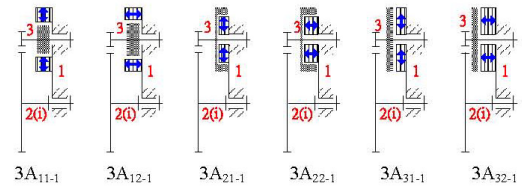

(a) Brushless gear dynamos

Figure 7. Structural sketches of the embedded three-link gear dynamos

Figure 8. Structural sketches of the embedded four-link gear dynamos 


\subsection{Induced magnetic circuits of the revolute joint in the dynamos}

The six induced magnetic circuits are labeled on the revolute joint of the dynamo. Since each configuration has two magnetic circuits on it, two induced magnetic circuits of the exterior-rotor configuration, $\mathrm{Cr}-\mathrm{Gr}$ and $\mathrm{Ca}-\mathrm{Gr}$, are labeled to graph $4 \mathrm{~B}_{2}$ in Figure 4 (d) and graph $4 \mathrm{~B}_{21}$ in Figure $4(\mathrm{e})$ is obtained. Finally, by applying the same process, 78 $(=39 \times 2)$ embedded four-link gear dynamos with induced magnetic circuit are obtained as shown in Figure 4(f). For another three-link example, two induced magnetic circuits are labeled to graph $3 \mathrm{~A}_{1}$ in Figure $6(\mathrm{~b})$ and graphs $3 \mathrm{~A}_{11}$ and $3 \mathrm{~A}_{12}$ in Figure 6(c) are obtained. Following the same process, there are $12(=6 \times 2)$ three-link graphs as shown in Figure 6(c).

\subsection{Elimination of existing designs}

The topological structures of existing embedded four-link gear dynamos are fixed stators within armature without brush and rotating rotors within permanent magnets. Now, permanent magnets and armatures are assigned to the two members of the dynamo in Figure 4(e). Referring to the proposed definitions of graph representations, these 12 graphs can be transferred to $24(=12 \times 2)$ structural sketches after the assignment of the permanent magnet and the armature, Figure 7 (a) shows the structural sketches of the embedded three-link gear brushless dynamo that the stator is assigned as the armature and the rotor as the permanent magnet. In the structural representations, the fixed link, permanent magnet, the armature, and the induced magnetic circuit in armature are expressed and defined as shown in Table 7. The direction of the air gaps is determined between the armature and the permanent magnet with respect to the rotation axis. The direction of the coil windings is also determined by the direction of the arrow respect to the rotation axis.

For structure $3 \mathrm{~A}_{11-1}$ in Figure $7(\mathrm{a})$, link 1 is a fixed stator with armature, link 2 is the input link with an external gear, and link 3 is an external gear within a permanent magnet for an interior-rotor type configuration. Furthermore, the direction of the coil windings is radial, and the direction of the air gaps is radial. Structures $3 \mathrm{~A}_{12-1}, 3 \mathrm{~A}_{21-1}, 3 \mathrm{~A}_{22-1}, 3 \mathrm{~A}_{31-1}$, and $3 \mathrm{~A}_{32-1}$ in Figure $7(\mathrm{a})$ are the six existing mechanisms with brushless dynamo to transmit the electricity to the fixed member. And the six structures $3 \mathrm{~A}_{11-2}, 3 \mathrm{~A}_{12-2}, 3 \mathrm{~A}_{21-2}, 3 \mathrm{~A}_{22-2}$, $3 \mathrm{~A}_{31-2}$, and $3 \mathrm{~A}_{32-2}$ in Figure $7(\mathrm{~b})$ are also the existing mechanisms with brush dynamo to transmit the electricity to the fixed member. Excluding the above 12 existing mechanisms, there are 12 novel embedded three-link gear brush dynamos. As a result, after designating the armatures on stators and rotors, there are two types of embedded gear brushless and brush dynamos, respectively.

The first type of the embedded gear brushless dynamos is the fixed stator with a permanent magnet and the rotating rotor with an armature. After assigning the stator with an armatures and the rotor with a permanent magnet to the dynamo, 13 graphs $\left(4 \mathrm{~B}_{21}, 4 \mathrm{~F}_{21}\right.$, $4 \mathrm{~J}_{21}, 4 \mathrm{~L}_{21}, 4 \mathrm{~N}_{21}, 4 \mathrm{O}_{21}, 4 \mathrm{Q}_{21}, 4 \mathrm{R}_{21}, 4 \mathrm{~S}_{21}, 4 \mathrm{~T}_{21}, 4 \mathrm{U}_{21}, 4 \mathrm{~W}_{21}$, and $4 \mathrm{Y}_{21}$ ) are obtained in Figure 4(e). According to the proposed structural representations, these 13 graphs are transferred to the structural sketches as shown in Figure 8. However, once these 13 graphs are assigned the stator with a permanent magnet and the rotor with an armature, they are the second type embedded four-link gear brush dynamos. This type also includes one rotating rotor with a permanent magnet and another rotating rotor with an armature. So, armatures and permanent magnets are assigned to the graphs shown in Figure 4(e). And, the permanent magnets and armatures on the two rotors are interchangeable. As a result, there are $26(=13 \times 2)$ structures of embedded four-link gear brush dynamos. However, structures $4 \mathrm{~F}_{21-1}$ and $4 \mathrm{~F}_{21-2}$ in Figure $8(\mathrm{~b})$ are two existing mechanisms. Finally, there are 4 novel embedded four-link gear brushless dynamos and 20 novel embedded four-link gear brush dynamos. 


\section{Prototypes}

According to the definitions of the graphs and structures, the embedded gear dynamos can be constructed. And, from the synthesized and sketched results in Figures 7 and 8 , the concepts of the integrated designs are modeled. In what follows, two prototypes with three-link and four-link gear dynamos are built.

\subsection{An embedded three-link gear dynamo}

From the structure $3 \mathrm{~B}_{11-2}$ in Figure 7(b), a prototype of embedded three-link gear brush dynamo with two rotors is presented as shown in Figure 9(a). The dynamo of case III in Figure 3 is modified to embed with the three-link plenary gear train. However, the stator is not the fixed member here and becomes the rotor with relative rotation to another rotor. Link 1 is the carrier and the input link within a rotor with a permanent magnet on it. Link 2 is the sun gear and the fixed link. Link 3 is the plenary gear combining with the shaft of another rotor and the armature is also on the same member. As shown in Figure 5, the corresponding induced magnetic circuit is represented as $\mathrm{Cr}-\mathrm{Gr}$. Since the mechanism rotates, the carrier continuously drives it due to its larger inertia. This design has fewer components and has no crank or ratchet gear to drive the mechanism. And, the case III dynamo generates the electricity to light the LED bulb on the carrier without additional brush and the rotational motion is continuous during the generation period. This continual generated current is much more than the conventional design.

\subsection{An embedded four-link gear dynamo}

From the structure $4 \mathrm{U}_{21-2}$ in Figure 8, a prototype concept of embedded four-link gear brushless dynamo with a stator and a rotor is presented as shown in Figure 9(b). The dynamo of case I in Figure 3 is modified to embed with the four-link plenary gear train. However, the permanent magnet is the fixed stator, and the armature is the rotating rotor. Link 1 is the plenary compound gear. Link 2 is a sun gear within an armature of interior-rotor configuration. Link 3 is the carrier to drive the mechanism as an input link. Link 4 is the fixed link within a permanent magnet and another sun gear. The corresponding induced magnetic circuit is represented as $\mathrm{Ca}-\mathrm{Gr}$ as shown in Figure 3. Since the mechanism rotates, the gear train drives the dynamo and it generates the conducted current to light the LED bulb on the sun gear without additional brush. In addition, the mechanism is driven by the additional large inertia of the plenary compound gear without a crank or ratchet gear. As a result, the mechanism is compact and simple for the continuous rotation during the power generation period. This continual generated current is much more than the conventional design. 


\section{Conclusions}

Based on the presented graph representations and topology and motion characteristics, this work proposes a systematic design methodology for the conceptual design of embedded dynamos with gear trains. The transformation between graphs and structures are defined. The relative motion of embedded gear dynamos with a two-rotors type is used for novel configurations by the larger inertia of the carrier as the input link. All possible three-link and four-link configurations are obtained and classified as embedded gear dynamos with or without brush, respectively. Furthermore, the speed reduction ratios of three-link and four-link configurations are derived for designers to select the applicable mechanisms. Four types of induced magnetic circuits are applied to each configuration of the three-link gear dynamo. Finally, the permanent magnet and the armature are designated on the members with their corresponding induced magnetic circuit. This provides a solid foundation for the detail design and construction of the embedded gear dynamos, and also provides a feasible approach for generating the integrated configurations of electrical motors and gear transmissions.

\section{Acknowledgements}

The authors are grateful to the National Science Council (TAIWAN, R.O.C.) for supporting this research under grant NSC94-2212-E-006-056.

\section{References}

(1) Vetorino, S.R., Platt, J.V., Springer, D.A., Renewable Energy Flashlight, (2001), US Patent 6,220,719.

(2) Choe, P.Y., Bench-Type Mechanical Light Generating Device, (1982), US Patent $4,332,006$.

(3) Johnson, H.G., Trattner, B.C., Self-Contained Hand Held Portable Lantern Flashlight Consisting of a Manually Operated Generator and Rechargeable Batteries, (1982), US Patent 4,360,860.

(4) Campagnuolo, C.J., Benderly, A.A., Multimode Flashlight, (1987), US Patent $4,701,835$.

(5) Millar, M.A., Non-Battery Powered Portable Lamp, (2003), US Patent 6,588,918.

(6) Jimena, C.I., Generator Flashlight, (1982), US Patent 4,315,301.

(7) Wei, M.C., Dynamo-Powered Torch, (1998), US Patent 5,839,817.

(8) Frankie, E.L., Pan Self Unit Portable Alternator Nerve Stimulator. (2002) US Patent $6,309,407$.

(9) Modernoutpost (2005) Gear Index, Lights \& Lanterns, http://www.modernoutpost.com/

(10) Yan, H.S., Wang, H.T., Liu, J.Y., Structural synthesis of novel integrated DC gear motors. Mechanism and Machine Theory, Vol. 41, (2006), pp.1289-1305.

(11) Harary, F., Yan, H.S., Logical foundations of kinematic chains: graphs, line graphs, and hypergraphs. ASME Transactions, Journal of Mechanical Design, Vol. 112, No. 1, (1990), pp.79-83.

(12) Bushsbaum, F., Freudenstein, F., Synthesis of kinematic structure of geared kinematic chains and other mechanisms. Journal of Mechanisms, No. 5, (1970), pp.357-392.

(13) Yan, H.S., Creative Design of Mechanical Devices, (1998), Springer, Singapore, pp.97-115, 159-169.

(14) Hsu, C.H., Lin, Y.L., Automatic analysis of the redundant gears in planetary gear trains. International Journal of Vehicle Design, No.15, Vol. 3/4/5, (1994), pp.402-415.

(15) Tsai, L.W., Mechanism Design: Enumeration of Kinematic Structures According to Function, (2001), CRC press, Boca Raton, Florida, pp.162-172. 